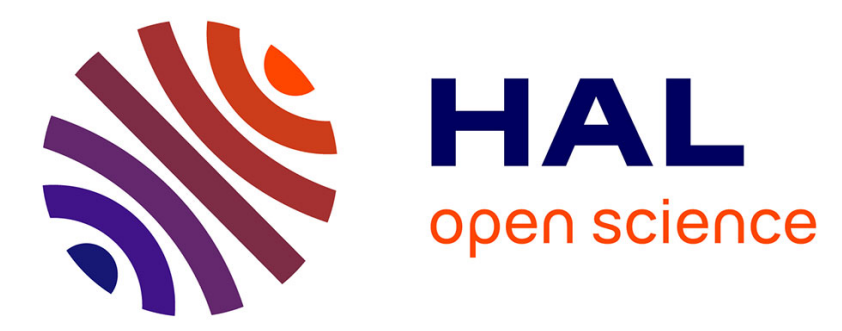

\title{
A triangular canonical form for a class of 0-flat nonlinear systems
}

\author{
Driss Boutat, Soray Bououden, Driss Boutat, Gang Zheng, Jean-Pierre \\ Barbot, Frédéric Kratz
}

\section{- To cite this version:}

Driss Boutat, Soray Bououden, Driss Boutat, Gang Zheng, Jean-Pierre Barbot, et al.. A triangular canonical form for a class of 0-flat nonlinear systems. International Journal of Control, 2011, 84 (2), pp.261-269. 10.1080/00207179.2010.549844 . hal-00627655

\section{HAL Id: hal-00627655 \\ https://hal.science/hal-00627655}

Submitted on 29 Sep 2011

HAL is a multi-disciplinary open access archive for the deposit and dissemination of scientific research documents, whether they are published or not. The documents may come from teaching and research institutions in France or abroad, or from public or private research centers.
L'archive ouverte pluridisciplinaire HAL, est destinée au dépôt et à la diffusion de documents scientifiques de niveau recherche, publiés ou non, émanant des établissements d'enseignement et de recherche français ou étrangers, des laboratoires publics ou privés. 


\title{
A triangular canonical form for a class of 0 -flat nonlinear systems
}

\author{
S. Bououden ${ }^{\mathrm{a}}$, D. Boutat ${ }^{\mathrm{a}}$, G. Zheng ${ }^{\mathrm{b} *}$, J.-P. Barbot $^{\mathrm{bc}}$ and F. Kratz \\ ${ }^{a}$ Loire Valley University, ENSI de Bourges, Institut PRISME, 88 Boulevard Lahitolle, \\ 18020 Bourges Cedex, France; ' INRIA, 40 Avenue Halley, Villeneuve d'Ascq 59650, \\ France; ${ }^{c} E C S / E N S E A, 6$ Avenue du Ponceau, Cergy-Pontoise 95014, France
}

(Received 11 November 2010; final version received 17 December 2010)

\begin{abstract}
This article proposes a triangular canonical form for a class of 0 -flat nonlinear systems. Necessary and sufficient geometrical conditions are given in order to guarantee the existence of a local diffeomorphism to transform the studied nonlinear systems into the proposed 0 -flat canonical form, which enables us to compute the flat output as well.
\end{abstract}

Keywords: flatness; canonical form; geometrical condition

\section{Introduction}

Differential flatness is a well-known concept in control theory, proposed by Fliess, Lévine, Martin and Rouchon (Fliess, Lévine, Martin, and Rouchon 1992, 1993, 1995, 1997, 1998, 1999a,b; Sira-Ramirez and Agrawal 2004; Lévine 2009) and nowadays is widely used in industrial applications, such as trajectory planning problem (Rotella and Zambettakis 2007). Other problems, such as isolation of the faults, left invertibility for nonlinear systems, synchronisation of chaotic systems, sometimes can be seen as a problem whether the system is flat (Pomet 1995a; Tilbury, Murray, and Sastry 1995; van Nieuwstadt, Rathinam, and Murray 1998; Hagenmeyer and Delaleau 2003; Lévine 2004; Schlacher and Schöberl 2007).

In fact, flat systems are a generalisation of linear dynamical systems in the sense that all linear controllable dynamical systems are flat and static feedback linearisable (in Brunovsky's form) (Jakubczyk and Respondek 1980; Singh 1981; Shadwick 1990; Sluis 1993; Shadwick and Sluis 1994; Rouchon 1995; Rudolph 1995; Sluis and Tilbury 1996). In contrast to the feedback linearisation techniques, the property of flatness does not need to convert nonlinear systems into linear ones to design different kinds of feedback laws (Hagenmeyer and Delaleau 2003; Lévine 2004; Schlacher and Schöberl 2007). Therefore, when a system is flat, one can use its structure to design control for motion, trajectory generation and stabilisation.

In order to apply the concept of flatness, one key problem is to determine the flat outputs for nonlinear systems. For this, many approaches have been proposed in the literature. One approach to deal with flatness is exterior differential systems where a control dynamical system is regarded as a Pfaffian system on an appropriate jet space (Anderson and Ibragimov 1979; Descusse and Moog 1985; Pomet 1995a; Delaleau and da Silva 1998; van Nieuwstadt et al. 1998; da Silva 2000a), and flatness is related to absolute equivalence introduced by Cartan (1914). Another geometrical approach by means of Lie-Bäcklund equivalence was addressed in Fliess et al. (1993, 1997, 1998) and Martin, Murray, and Rouchon (1997). In addition, since feedback linearisable dynamical systems are flat, some results in this direction are stated, such that controllable codimension 1 affine dynamical systems. In da Silva (2000) authors gave a characterisation of the so-called $k$-flatness with the Cartan-Kähler approach. There are also many interesting results on flatness of driftless dynamical systems. We can cite the work of Martin and Rouchon (1995a,b) where the authors proved that any (controllable) driftless system with $m$ inputs and $m+2$ states is flat. Another interesting result on 1-flatness can be found in Pomet (1995b) for fourdimensional dynamical systems.

However, for general nonlinear systems, it is still an open problem to construct flat outputs. As an extension of Bououden, Boutat, Barbot, and Kratz (2009), this article studies this problem from normal form point of view, by proposing a triangular 0-flat canonical form for a class of nonlinear systems, whose outputs are flat. Some results on triangular flat forms are reported by using states transformation and static states feedback. For instance, for five states and two controls, every 0-flat system can be transformed into a

*Corresponding author. Email: gang.zheng@inria.fr

ISSN 0020-7179 print/ISSN 1366-5820 online

(C) 2011 Taylor \& Francis

DOI: $10.1080 / 00207179.2010 .549844$

http://www.informaworld.com 
triangular form by state transformation and regular static-state feedback (Silveira and da Silva 2007). Other results about the relationship between triangular forms and flatness can be found in Silveira (2009). It has been proved that a system can be $k$-flat but not $(k-1)$-flat and an example, which is not 0-flat but 1-flat, is given in da Silva (2000b). It should be noted that we treat the linearisation problem only through state transformations without static states feedback in this article. Necessary and sufficient geometrical conditions are given in order to guarantee the existence of a local diffeomorphism to transform the studied nonlinear systems into the proposed 0-flat canonical form.

This article is organised as follows. Notations and definitions are given in Section 2. In Section 3, a class of triangular 0-flat canonical form is proposed and its associated geometrical interpretation is discussed. Section 4 presents the necessary and sufficient geometrical conditions to locally transform nonlinear systems into the proposed 0-flat canonical form.

\section{Notations and definitions}

Let us first recall the notion of flatness of the nonlinear system of the following form:

$$
\dot{x}=f(x, u),
$$

where $x \in \mathcal{X} \subseteq \mathbb{R}^{n}, u \in \mathcal{U} \subseteq \mathbb{R}^{m}$ and $f$ is assumed to be a smooth vector field on $\overline{\mathcal{X}} \times \mathcal{U}$.

Definition 2.1: Dynamical system (1) is flat if there exist $m$ functions $y=\left(y_{1}, \ldots, y_{m}\right)^{T}$, called flat outputs, where $m$ is the number of inputs, such that

(1) the flat output is a unique function of the state $x$, the input $u$ and its derivatives $u^{(i)}$, i.e. $y\left(x, u, \dot{u}, \ldots, u^{\left(r_{1}\right)}\right)$.

(2) the state can be written as a function of the flat output and its derivatives, i.e. $x=$ $\varphi\left(y, \dot{y}, \ldots, y^{\left(r_{2}\right)}\right)$.

(3) the input can be written as a function of the flat output and its derivatives, i.e. $u=$ $\gamma\left(y, \dot{y}, \ldots, y^{\left(r_{2}+1\right)}\right)$.

Without loss of generalities, in this article we are interested in affine dynamical systems in the following form:

$$
\dot{x}=f(x)+\sum_{i=1}^{m} g_{i}(x) u_{i} .
$$

Remark 1: A system of the form (1) can be brought into the form (2) by adding an integrator to each input.

The objective of this article is to characterise a class of dynamical systems for which the flat outputs are only functions of states $x$. This implies that in (1) of Definition 2.1 we have only $y(x)$. In the literature, these systems are called 0-flat (da Silva 2000a).

Let us denote for $i=1: m$ by $a d_{f} g_{i}$ the Lie bracket of $f$ with $g_{i}$, i.e.

$$
a d_{f} g_{i}=\left[f, g_{i}\right]
$$

and by induction we have $a d_{f}^{k} g_{i}=\left[a d_{f}^{k-1} g_{i}, g_{i}\right]$ for $0 \leq k$ with conventional notation $a d_{f}^{0} g_{i}=g_{i}$. It is well-known that, if (2) can be linearised by a diffeomorphism $z=\phi(x)$ and a static states feedback $u=\alpha(x)+\beta(x) v$ into the following form:

$$
\begin{aligned}
& \dot{z}_{i, j}=z_{i+1, j}, \quad \text { for } 1 \leq j \leq m \text { and } 1 \leq i \leq v_{j}-1, \\
& \dot{z}_{v_{j, j}}=v_{j}
\end{aligned}
$$

with $\sum_{j=1}^{m} v_{j}=n$, then $y_{j}=z_{1, j}$ for $1 \leq j \leq m$ are the 0 -flat outputs of (2). To transform a dynamical system into the form (3) is called the static states feedback linearisation problem and we have the following famous result (see e.g. Jakubczyk and Respondek 1980; Hunt, Su, and Meyer 1983).

Theorem 2.2: The linearisation problem for (2) can be solved if and only if

(1) the dimension of $\mathcal{G}_{i}$ is constant on $\mathcal{X}$ for $1 \leq i \leq n-1$,

(2) the dimension of $\mathcal{G}_{n-1}$ is of $n$,

(3) the distribution $\mathcal{G}_{k}$ is involutive for each $1 \leq k \leq n-2$,

where

$$
\begin{aligned}
& \mathcal{G}_{0}=\operatorname{span}\left\{g_{1}, \ldots, g_{m}\right\}, \\
& \mathcal{G}_{1}=\operatorname{span}\left\{g_{1}, \ldots, g_{m}, a d_{f} g_{1}, \ldots, a d_{f} g_{m}\right\}, \\
& \quad \vdots \\
& \mathcal{G}_{k}=\operatorname{span}\left\{a d_{f}^{j} g_{i}, \text { for } 0 \leq j \leq k, 1 \leq i \leq m\right\} .
\end{aligned}
$$

Another famous class of 0 -flat dynamical systems is controllable affine systems with $n$ states and $n-1$ inputs (Martin et al. 1997; Rotella and Zambettakis 2007). In what follows, we will propose a more general triangular canonical form than (3) and prove that it is 0 -flat. Then we will give sufficient and necessary geometrical conditions to guarantee the existence of a local diffeomorphism which transforms (2) into the proposed canonical form.

\section{A class of 0-flat dynamical system}

This section first gives a cascade 0-flat normal form which represents a class of affine flat dynamical systems, then gives a geometrical characterisation of 
such a normal form. It should be noted that the proposed conditions are only sufficient and the considered dynamical systems are only a small class. Therefore there exists many dynamical systems, in particular flat driftless systems do not check the proposed conditions, and examples can be found in Martin and Rouchon (1995a,b) and Pomet (1995b).

\subsection{Triangular 0-flat canonical form}

In this section, we give a class of 0 -flat dynamical systems, represented by its normal form, which generalises the well-known controllable affine systems with $n$ states and $n-1$ inputs. For this, it is assumed that there exists a list of integers:

$$
v_{1} \geq v_{2} \geq \cdots \geq v_{m}
$$

such that $\sum_{j=1}^{m} v_{j}=n$.

Now, for all $1 \leq j \leq m$, denote $z_{j}=\left(z_{1, j}, \ldots, z_{v_{j}, j}\right)^{T}$ and define the following system:

$\dot{z}_{i, j}=z_{i+1, j}+\sum_{l=1}^{m} \alpha_{i, j}^{l}(z) u_{l}$ for $1 \leq j \leq m, 1 \leq i \leq v_{j}-1$,

$$
\dot{z}_{v_{j, j}}=a_{j}(z)+\sum_{l=1}^{m} \alpha_{v_{j}, j}^{l}(z) u_{l}
$$

where $\alpha_{i, j}^{l}(z)$ and $a_{j}(z)$ satisfy the following property.

\section{Property 3.1:}

(1) Functions $\alpha_{i, j}^{l}$ for $1 \leq l \leq m, 1 \leq j \leq m$ and $1 \leq i \leq v_{j}$ are as follows:

- $\alpha_{v_{j}, j}^{j} \neq 0$ on $\mathcal{X}$;

- if $v_{l}>i$, then $\alpha_{i, j}^{l}=0$;

- if $v_{l} \leq i$, then $\alpha_{i, j}^{l}$ depend only on $z_{s, k}$ for $1 \leq k \leq m$ and $1 \leq s \leq \min \left\{i+1, v_{k}\right\}$.

(2) Functions $a_{j}$ for $1 \leq j \leq m$ depend only on the following variables:

- $z_{i, k}$ for $k \in\left\{k \in[1, m] \mid v_{k}>v_{j}\right\}$ and $1 \leq i \leq$ $v_{j}+1$

- $z_{i, k}$ for $k \in\left\{k \in[1, m] \mid v_{k} \leq v_{j}\right\}$ and $1 \leq i \leq v_{k}$.

Remark 2: According to the form (4), (5), for $1 \leq j \leq m$, the dynamics $\dot{z}_{1, j}$ depends only on $u_{l}$ for $l \in\left\{l \in[1, m] \mid v_{l}=1\right\}, \quad z_{1, k} \quad$ for $\quad 1 \leq k \leq m$ and $z_{2, k}$ for $k \in\left\{k \in[1, m] \mid v_{k} \geq 2\right\}$. For $1 \leq j \leq m$, the dynamics $\dot{z}_{2, j}$ depends only on $u_{l}$ for $l \in\left\{l \in[1, m] \mid v_{l} \leq 2\right\}, z_{1, k}$ for $1 \leq k \leq m, \quad z_{2, k}$ for $1 \leq k \leq m$ and $z_{3, k}$ for $k \in\{k \in$ $\left.[1, m] \mid v_{k} \geq 3\right\}$.
By induction, it can be seen that the dynamics $\dot{z}_{i, j}$ for $1 \leq j \leq m$ and $1 \leq i \leq v_{j}$ depend on the following variables:

- $u_{l}$ for $l \in\left\{l \in[1, m] \mid v_{l} \leq i\right\}$;

- $z_{s, k}$ for $1 \leq k \leq m$ and $1 \leq s \leq \min \left\{i+1, v_{k}\right\}$,

which implicitly yield a triangular structure allowing to calculate $z_{i+1, j}$ for $1 \leq j \leq m$ and $1 \leq i \leq v_{j}-1$ and $u_{l}$ for $1 \leq l \leq m$ step by step.

Remark 3: By using a linear change of coordinates, we can always assume that $a_{j}$ for $1 \leq j \leq m$ contains only the terms with orders greater than 1 , i.e. $O^{2}(z)$, and $\alpha_{i, j}^{l}$ for $1 \leq l \leq m, 1 \leq j \leq m$ and $1 \leq i<v_{j}$ is of $O^{1}(z)$.

Remark 4: By setting $\alpha_{v_{j}, j}^{l}=1, \quad a_{j}=0 \quad$ and $\alpha_{v_{j}, j}^{l}=0$ for $i \neq v_{j}$, we see that the form (3) is a special case of the form (4), (5).

\subsection{Geometrical interpretation}

Before giving a geometrical interpretation of the above conditions, let us rewrite dynamical system (4), (5) into the following compact form:

$$
\dot{z}=\bar{f}+\sum_{k=1}^{m} \bar{g}_{k} u_{k}
$$

with

$$
\bar{f}=\operatorname{col}\left(\bar{f}_{1}, \bar{f}_{2}, \ldots, \bar{f}_{m-1}, \bar{f}_{m}\right)
$$

and

$$
\bar{f}_{j}=\operatorname{col}\left(z_{2, j}, z_{3, j}, \ldots, z_{v_{j}, j}, a_{j}\right)
$$

for $1 \leq j \leq m$, and

$$
\bar{g}_{l}=\operatorname{col}\left(\bar{g}_{1}^{l}, \bar{g}_{2}^{l}, \ldots, \bar{g}_{m}^{l}\right)
$$

and

$$
\bar{g}_{j}^{l}=\operatorname{col}\left(\alpha_{1, j}^{l}, \alpha_{2, j}^{l}, \ldots, \alpha_{v_{j}, j}^{l}\right)
$$

for $1 \leq l \leq m$ and $1 \leq j \leq m$.

Since $\alpha_{i, j}^{l}$ for $1 \leq l \leq m, 1 \leq j \leq m$ and $1 \leq i \leq v_{j}$ satisfies Property 3.1 , for $1 \leq l \leq m$ we have

$$
\bar{g}_{j}^{l}=0 \quad \text { if } v_{j}<v_{l},
$$

which gives the geometrical characteristic of (4), (5) in the compact form (6) as follows.

Proposition 3.2: The canonical form (4), (5) in the compact form (6) satisfies the following conditions:

(1) The distribution

$$
\Delta=\operatorname{span}\left\{a d_{f}^{k} g_{j} \text { for } 1 \leq j \leq m, 0 \leq k \leq v_{j}-1\right\}
$$


is of dimension $n$. In this case we say that (2) is controllable and $v_{j}$ for $1 \leq j \leq m$ are the controllability indices. ${ }^{1}$

(2) The distribution $\bar{\Delta}$ defined as follows:

$$
\begin{gathered}
\bar{\Delta}=\operatorname{span}\left\{a d_{\bar{f}}^{k} \bar{g}_{i}, \text { for } j \in\left\{j \in[1, m] \mid v_{j} \geq 2\right\},\right. \\
\left.0 \leq k \leq v_{j}-2\right\}
\end{gathered}
$$

is involutive.

(3) The item (2) of Property 3.1 is equivalent to the following condition:

$$
\begin{aligned}
& {\left[\bar{g}_{l}, a d_{\bar{f}}^{v_{k}-v_{l}-1-s} \bar{g}_{k}\right] \in \operatorname{span}\left\{a d_{\bar{f}}^{i} \bar{g}_{j} \text { for } 0 \leq j \leq v_{i}-v_{l}-s\right.} \\
& \text { and } \left.j \in\left\{j \in[1, m] \mid v_{j} \geq v_{l}+s\right\}\right\}, \\
& \quad \text { where } k \in\left\{k \in[1, m] \mid v_{k} \geq 2\right\}, \quad l \in\{l \in[1, m] \mid \\
& \left.\quad v_{l}<v_{k}\right\} \text { and } 0 \leq s \leq v_{k}-v_{l}-1 .
\end{aligned}
$$

Proof: According to the triangular structure of (4), (5), condition (1) of Proposition 3.2 is easy to be proved. Moreover, the structure of (4), (5) allows to calculate $\bar{g}_{l}$ for $1 \leq l \leq m$, and by some straightforward calculations one can check that (3) of Proposition 3.2 is evident, and thus we only prove here (2) of Proposition 3.2.

Denote by $\bar{\Delta}^{\perp}$ the annihilator of $\bar{\Delta}$. According to the form (6) with Property 3.1, it can be proved that

$$
\bar{\Delta}^{\perp}=\operatorname{span}\left\{d z_{1, j}, \text { for } 1 \leq j \leq m\right\},
$$

which is spanned by the exact differential forms. Thus according to Frobenius Theorem, the distribution $\bar{\Delta}$ is involutive.

Then we have the following preliminary result.

Proposition 3.3: Dynamical system (4), (5) is locally 0 -flat and the variables $z_{1, j}$ for $1 \leq j \leq m$ are the flat outputs.

Proof: As mentioned in Remark 2, we can obtain a triangular structure to calculate the states and inputs step by step. For this, denote $\mathfrak{\Xi}_{s}$ for $1 \leq s \leq$ $\max \left\{v_{j}, 1 \leq j \leq m\right\}$ the following set of dynamics:

$$
\mathfrak{\Im}_{s}=\left\{\dot{z}_{i, j}, 1 \leq j \leq m \text { and } 1 \leq i \leq \min \left\{s, v_{j}\right\},\right.
$$

which in fact depends only on the following variables:

- $u_{l}$ for $l \in\left\{l \in[1, m] \mid v_{l} \leq i\right\}$;

- $z_{s, k}$ for $1 \leq k \leq m$ and $1 \leq s \leq \min \left\{i+1, v_{k}\right\}$;

and specially we denote $\mathfrak{\Xi}_{0}=\left\{z_{1, j}, 1 \leq j \leq m\right\}$ as the set of the flat outputs, thus we have $\Xi_{1}=\left\{\dot{z}_{1, j}\right.$, $1 \leq j \leq m\}=\left\{\xi, \xi \in \dot{\Xi}_{0}\right\}$.

Now we will show that $y_{j}=z_{1, j} \in \boldsymbol{\Xi}_{0}$ for $1 \leq j \leq m$ are the 0-flat outputs. First let us compute the following variables:

- $z_{2, j}$ for $v_{j} \geq 2$,

- $u_{l}$ for $v_{l}=1$ from the flat outputs and their derivatives. According to the form (4), (5),we have

$$
\begin{aligned}
& \dot{z}_{1, j}-z_{2, j}-\sum_{l=1}^{m} \alpha_{i, j}^{l} u_{l}=0 \quad \text { for } j \in\left\{j \in[1, m] \mid v_{j} \geq 2\right\}, \\
& \dot{z}_{1, j}-a_{j}-\sum_{l=1}^{m} \alpha_{i, j}^{l} u_{l}=0 \quad \text { for } j \in\left\{j \in[1, m] \mid v_{j}=1\right\},
\end{aligned}
$$

where $a_{j}$ and $\alpha_{1, j}^{l}$ satisfy Property 3.1, and thus depend only on the known variable $z_{1, k}$ for $1 \leq k \leq m$ and the unknown variables $u_{k}$ for $k \in\left\{k \in[1, m] \mid v_{k}=1\right\}$ and $z_{2, k}$ for $k \in\left\{k \in[1, m] \mid v_{k} \geq 2\right\}$.

So (8) is formed with $m$ equations and contains $m$ unknown variables. According to Property 3.1 and Remark 3, partial differentiation of the left-hand side of (8) with respect to $\frac{\partial}{\partial\left(z_{2, j}, u_{k}\right)}$ is equal to $I+O^{1}(z, u)$. Therefore, thanks to the implicit function theorem one can find $m$ functions: $\varphi_{j}$ for $v_{j} \geq 2$ and $\gamma_{l}$ for $v_{l}=1$ such that

$$
\begin{aligned}
z_{2, j}=\varphi_{j}\left(z_{1, k}, \dot{z}_{1, k}\right) & \text { for } 1 \leq k \leq m, \\
u_{l}=\gamma_{l}\left(z_{1, k}, \dot{z}_{1, k}\right) & \text { for } 1 \leq k \leq m .
\end{aligned}
$$

Thus it can be stated that

$$
\begin{aligned}
& z_{2, j} \in\left\{\xi, \xi \in \Xi_{0} \cup \Xi_{1}\right\} \text { for } j \in\left\{j \in[1, m] \mid v_{j} \geq 2\right\}, \\
& u_{l} \in\left\{\xi, \xi \in \Im_{0} \cup \Im_{1}\right\}, \quad \text { for } l \in\left\{l \in[1, m] \mid v_{l}=1\right\}
\end{aligned}
$$

and $\widetilde{\Xi}_{2}=\left\{\xi, \xi \in \dot{\Xi}_{0} \cup \ddot{\Xi}_{0}\right\}$.

Now, similar to the first step, for the following variables:

- $z_{3, j}$ for $v_{j} \geq 3$,

- $u_{l}$ for $v_{l}=2$,

one can obtain

$$
\begin{aligned}
z_{3, j} & =\varphi_{j}\left(z_{1, k}, \dot{z}_{1, k}, z_{2, k}, \dot{z}_{2, k}\right), \\
u_{l} & =\gamma_{l}\left(z_{1, k}, \dot{z}_{1, k}, z_{2, k}, \dot{z}_{2, k}\right),
\end{aligned}
$$

which implies that

$$
\begin{aligned}
& z_{3, j} \in\left\{\xi, \xi \in \widetilde{\Xi}_{0} \cup \dot{\Xi}_{0} \cup \ddot{\Xi}_{0}\right\} \text { for } j \in\left\{j \in[1, m] \mid v_{j} \geq 3\right\} \text {, } \\
& u_{j} \in\left\{\xi, \xi \in \Xi_{0} \cup \dot{\Xi}_{0} \cup \ddot{\Xi}_{0}\right\} \quad \text { for } j \in\left\{j \in[1, m] \mid v_{j}=2\right\}
\end{aligned}
$$

and $\mathfrak{\Xi}_{3}=\left\{\xi, \xi \in \cup_{i=1}^{3} \mathfrak{\Xi}_{0}^{(i)}\right\}$.

By induction, assume that for $1 \leq s<\max \left\{v_{j}\right.$, $1 \leq j \leq m\}$ we have computed $\mathfrak{\Xi}_{s}=\left\{\xi, \xi \in \sup _{i=1}^{s} \mathfrak{\Xi}_{0}^{(i)}\right\}$, and one has

$$
\begin{aligned}
z_{s+1, j} & \in\left\{\xi, \xi \in \cup_{i=1}^{s} \mathfrak{\Im}_{0}^{(i)}\right\} \quad \text { for } j \in\left\{j \in[1, m] \mid v_{j} \geq s\right\}, \\
& u_{l} \in\left\{\xi, \xi \in \cup_{k=1}^{s} \mathfrak{\Im}_{0}^{(k)}\right\} \text { for } l \in\left\{l \in[1, m] \mid v_{l}=s-1\right\} .
\end{aligned}
$$

Finally, we show that all states and inputs can be written as functions of $y_{1, j}$ for $1 \leq j \leq m$ and its derivatives, and thus prove Proposition 3.3. 
Let us give an example which is already in the 0-flat canonical form (4), (5), in order to show the procedure of computation of the state variables and the inputs proposed in the above proof.

Example 3.4: Consider the following dynamical system:

$$
\left\{\begin{array}{l}
\dot{z}_{1,1}=z_{2,1}, \\
\dot{z}_{2,1}=z_{3,1}+\frac{z_{2,1}}{z_{2,2}-1} u_{2}, \\
\dot{z}_{3,1}=\left(z_{2,2}-1\right) u_{1}+\frac{z_{3,1}}{z_{2,2}-1} u_{2} \\
\dot{z}_{1,2}=z_{2,2} \\
\dot{z}_{2,2}=u_{2}
\end{array}\right.
$$

and suppose that $y_{1}=z_{1,1}$ and $y_{2}=z_{1,2}$, we will give the procedure to compute all state variables and inputs from the outputs. For this, let us consider the following dynamics:

$$
\left\{\begin{array}{l}
\dot{z}_{1,1}-z_{2,1}=0 \\
\dot{z}_{1,2}-z_{2,2}=0
\end{array}\right.
$$

we obtain $z_{2,1}=\dot{y}_{1}$ and $z_{2,2}=\dot{y}_{2}$.

Let us again consider the derivatives of these obtained variables

$$
\left\{\begin{array}{l}
\dot{z}_{2,1}=z_{3,1}+\frac{z_{2,1}}{z_{2,2}-1} u_{2} \\
\dot{z}_{2,2}=u_{2}
\end{array}\right.
$$

which gives $u_{2}=\ddot{y}_{2}$ and $z_{3,1}=\ddot{y}_{1}-\frac{\dot{y}_{1}}{\dot{y}_{2}-1} \ddot{y}_{2}$.

Finally, from the third equation of (11) we obtain

$$
\dot{z}_{3,1}-\left(z_{2,2}-1\right) u_{1}-\frac{z_{3,1}}{z_{2,2}-1} u_{2}=0,
$$

which gives

$$
u_{1}=\frac{1}{\dot{y}_{2}-1}\left(y_{1}^{(3)}-\frac{\partial\left(\frac{\dot{y}_{1}}{\dot{y}_{2}-1} \ddot{y}_{2}\right)}{\partial t}-\frac{\ddot{y}_{1}-\frac{\dot{y}_{1}}{\dot{y}_{2}-1} \ddot{y}_{2}}{\dot{y}_{2}-1} \ddot{y}_{2}\right) .
$$

\section{Geometrical conditions to transform nonlinear systems into the proposed canonical form}

In this section, we will show that conditions (1)-(3) of Proposition 3.2 are sufficient for the existence of a local diffeomorphism which transforms an affine dynamical system of the form (2) into (4), (5).

For this, assume that for (2) there exist $m$ integers $v_{1} \geq v_{2} \geq \cdots \geq v_{m}$ such that

(1) $\sum_{i=1}^{m} v_{i}=n$

(2) $\Delta=\operatorname{span}\left\{a d_{f}^{k} g_{j}\right.$ for $1 \leq j \leq m$ and $\left.1 \leq k \leq v_{k}-1\right\}$ is of constant dimension $n$ on $\mathcal{X}$.
Let us also define the following distribution:

$\bar{\Delta}=\operatorname{span}\left\{a d_{f}^{k} g_{j}\right.$ for $\left.i \in\left\{i \in[1, m] \mid v_{j} \geq 2\right\}, 0 \leq k \leq v_{j}-2\right\}$,

then we are ready to give our main result.

Theorem 4.1: There exists a local diffeomorphism which transforms dynamical system (2) into the (4), (5) form if and only if

(1) $\bar{\Delta}$ defined in (12) is involutive;

$$
\begin{aligned}
& {\left[g_{l}, a d_{f}^{v_{k}-v_{l}-1-s} g_{k}\right] \in \operatorname{span}\left\{a d_{f}^{i} g_{j} \text { for } 0 \leq i \leq v_{j}-v_{l}-s\right.} \\
& \left.\quad \text { and } j \in\left\{j \in[1, m] \mid v_{j} \geq v_{l}+s\right\}\right\}
\end{aligned}
$$

where $k \in\left\{k \in[1, m] \mid v_{k} \geq 2\right\}, l \in\left\{l \in[1, m] \mid v_{l}<v_{k}\right\}$ and $0 \leq s \leq v_{k}-v_{l}-1$.

Proof: According to Proposition 3.2, the necessity of Theorem 4.1 is obvious. Thus we prove only the sufficiency. For this, without loss of, generality, assume that there exists $1 \leq r \leq m$ such that

$$
v_{j} \geq 2 \text { for } 1 \leq j \leq r
$$

and

$$
v_{j}=1 \text { for } r+1 \leq j \leq m .
$$

Thus, according to the definition of $\bar{\Delta}$ in (12), since $v_{r+1}+\cdots+v_{m}=m-r$, one has

$$
\begin{aligned}
\operatorname{dim} \bar{\Delta} & =v_{1}+\cdots+v_{r}-r \\
& =n-\left(v_{r+1}+\cdots+v_{m}\right)-r \\
& =n-m,
\end{aligned}
$$

which means that the codistribution $\bar{\Delta}^{\perp}$ has

$$
\operatorname{dim} \bar{\Delta}^{\perp}=m .
$$

If $\bar{\Delta}$ is involutive, then by Frobenius Theorem, there exists a set of functions $\left\{h_{1}, \ldots, h_{m}\right\}$ locally defined around some $x_{0} \in \mathcal{X}$, such that the set $\left\{d h_{1}, \ldots, d h_{m}\right\}$ is locally independent around $x_{0}$ and

$$
d h_{j}(\bar{\Delta})=0 \quad \text { for } 1 \leq j \leq m,
$$$$
d h_{j}\left(a d_{f}^{v_{k}-1} g_{j}\right) \neq 0 \quad \text { on } \mathcal{X} \quad \text { for } 1 \leq j \leq m, 1 \leq k \leq m \text {. }
$$

After having $h_{j}$ for $1 \leq j \leq m$, define the following new variables:

$$
z_{i, j}=L_{f}^{i-1} h_{j} \quad \text { for } 1 \leq i \leq v_{j} .
$$

Set $z_{j}=\operatorname{col}\left(z_{i, j}\right)$ for $1 \leq j \leq m$ and $1 \leq i \leq v_{j}$, and $z=\operatorname{col}\left(z_{j}\right)$ for $1 \leq j \leq m$ which then defines a local diffeomorphism, noted as $z=\phi(x)=\operatorname{col}\left(\phi_{i, j}\right)$ for $1 \leq j \leq m$ and $1 \leq i \leq v_{j}$ with $\phi_{i, j}=z_{i, j}=L_{f}^{i-1} h_{j}$.

Let us now analyse the expression of the system in the new coordinates. Denote $f=\phi_{*}(f)$ and $\bar{g}_{s}=\phi_{*}\left(g_{l}\right)$ 
for $1 \leq l \leq m$ with $\bar{g}_{l}=\operatorname{col}\left(\alpha_{j}^{l}\right)$ for $1 \leq j \leq m$ where $\alpha_{j}^{l}=\operatorname{col}\left(\bar{\alpha}_{i, j}^{l}\right)$ for $1 \leq i \leq v_{l}$.

First, according to the definition of the above diffeomorphism $z=\phi(x)$, it is easy to see that for $1 \leq j \leq m$ and $1 \leq i<v_{j}$ one has

$$
\phi_{i, j *} f=d L_{f}^{i-1} h_{j} f=L_{f}^{i} h_{j}=\phi_{i+1, j}=z_{i+1, j} .
$$

Moreover, by the involutivity condition, functions $a_{j}$ for $1 \leq j \leq m$ fulfil (2) of Property 3.1, thus $\phi_{*} f$ is in the form $\bar{f}$ of (4), (5).

By the definition of the diffeomorphism, for $1 \leq j \leq m, 1 \leq i \leq v_{j}$ and $1 \leq l \leq m$ we have

$$
\alpha_{i, j}^{l}=\left[\phi_{*}\left(g_{l}\right)\right]_{i, j}=d L_{f}^{i-1} h_{j} g_{l} .
$$

Since $\mathrm{d} h_{j}(\bar{\Delta})=0$ for $1 \leq j \leq m$, then for $l \in\left\{l \in[1, m] \mid v_{l} \geq 2\right\}, k \leq v_{l}-2$ and $1 \leq j \leq m$ one has

$$
d h_{j} a d_{f}^{k} g_{l}=d L_{f}^{k} h_{j} g_{l}=\alpha_{k+1, j}^{l}=0,
$$

which means that for $1 \leq j \leq m, 1 \leq i \leq v_{j}$ and $1 \leq l \leq m$, one obtains

$$
\alpha_{i, j}^{l}=0 \quad \text { if } v_{l}>i
$$

Moreover, because $\phi$ is a diffeomorphism, thus for $1 \leq l \leq m$ and $k \geq 0$ one has the following equality:

$$
\begin{aligned}
\phi_{*}\left(a d_{f}^{k} g_{l}\right) & =\phi_{*}\left[a d_{f}^{k-1} g_{l}, g_{l}\right]=\left[\phi_{*} a d_{f}^{k-1} g_{l}, \phi_{*} g_{l}\right] \\
& =\left[a d_{\bar{f}}^{k-1} \bar{g}_{l}, \bar{g}_{l}\right]=a d_{\bar{f}}^{k} \bar{g}_{l} .
\end{aligned}
$$

Thus, by applying the diffeomorphism $\phi$, the second condition of Theorem 4.1 yields

$$
\begin{aligned}
& {\left[\bar{g}_{l}, a d_{\bar{f}}^{v_{k}-v_{l}-1-s} \bar{g}_{k}\right] \in \operatorname{span}\left\{a d_{f}^{i} \bar{g}_{j} \text { for } 0 \leq i \leq v_{j}-v_{l}-s\right.} \\
& \left.\quad \text { and } j \in\left\{j \in[1, m] \mid v_{j} \geq v_{l}+s\right\}\right\},
\end{aligned}
$$

where $k \in\left\{k \in[1, m] \mid v_{k} \geq 2\right\}, l \in\left\{l \in[1, m] \mid v_{l}<v_{k}\right\}$ and $0 \leq s \leq v_{k}-v_{l}-1$. According to Proposition 3.2, this means that, for $1 \leq l \leq m, \phi_{*}\left(g_{l}\right)$ is transformed into $\bar{g}_{l}$ of the form defined in (4), (5).

Corollary 4.2: Suppose that indices $v_{j}$ and $v_{k}$ for $1 \leq j \leq m, 1 \leq k \leq m$ and $i \neq j$ satisfy $\left|v_{j}-v_{k}\right| \leq 1$. The dynamical system (2) can be transformed into (4), (5) if and only if the distribution $\bar{\Delta}$ defined in (12) is involutive.

Moreover, for any $1 \leq j \leq m$ and $1 \leq k \leq m$, if $v_{j}=v_{k}$, then the 0-flat canonical form (4), (5) can be written in the following simpler form:

$$
\left\{\begin{array}{l}
\dot{z}_{i, j}=z_{i+1, j} \quad \text { for } 1 \leq i \leq v_{j}-2, \\
\dot{z}_{v_{j-1}, j}=z_{v_{j}, j}+\sum_{l=1}^{m} \alpha_{v_{j-1}, j}^{l} u_{l}, \\
\dot{z}_{v_{j}, j}=a_{j}+\sum_{l=1}^{m} \alpha_{v_{j}, j}^{l} u_{l},
\end{array}\right.
$$

where $a_{j}$ and $\alpha_{i, j}^{l}$ satisfy Property 3.1.
In particular, a codimension 1 dynamical system, i.e. $m=n-1$, is flat (the well-known result in Charlet and Lévine (1989)).

Remark 5: If $v_{j} \leq 2$ for all $1 \leq j \leq m$, then there exists a local diffeomorphism which transforms dynamical system (2) into (4), (5) if and only if the distribution

$$
\bar{\Delta}=\left\{g_{j}, \text { for } j \in\left\{j \in[1, m] \mid v_{j}=2\right\}\right.
$$

is involutive, since the second condition of Theorem 4.1 is always fulfilled. In fact, it is a special case of Corollary 4.2.

Remark 6: For single input systems, i.e. $m=1$, we only need the first condition of Theorem 4.1 and this condition is equivalent to the condition for the linearisation problem by means of a diffeomorphism and a static feedback.

Remark 7: For the case of codimension 2, i.e. $m=n-2$, by reordering $g_{j}$ for $1 \leq j \leq m$ if necessary, we have the following two cases:

(1) $v_{1}=2$ and $v_{2}=2$;

(2) $v_{1}=3$.

The first case is similar to Remark 5, thus we need to check the involutivity of the distribution $\bar{\Delta}=\operatorname{span}\left\{g_{1}, g_{2}\right\}$.

For the second case, we have to check the following two conditions:

- the distribution $\bar{\Delta}=\left\{g_{1}, a d_{f} g_{1}\right\}$ is involutive;

- for all $2 \leq k \leq m,\left[g_{k}, g_{1}\right] \in \operatorname{span}\left\{g_{1}, a d_{f} g_{1}\right\}$.

Example 4.3: Consider the following academic example (da Silva 2000a) modified for a regularity question:

$$
\left\{\begin{array}{l}
\dot{x}_{1}=x_{2}+x_{4} x_{3}, \\
\dot{x}_{2}=x_{4}, \\
\dot{x}_{3}=x_{5}, \\
\dot{x}_{4}=u_{1}, \\
\dot{x}_{5}=u_{2} .
\end{array}\right.
$$

A simple calculation shows that distribution $\Delta$ is spanned by the following vector fields:

$$
\begin{aligned}
g_{1} & =\frac{\partial}{\partial x_{4}}, \\
a d_{f} g_{1} & =-\frac{\partial}{\partial x_{2}}-x_{3} \frac{\partial}{\partial x_{1}}, \\
a d_{f}^{2} g_{1} & =\left(1-x_{5}\right) \frac{\partial}{\partial x_{1}}, \\
g_{2} & =\frac{\partial}{\partial x_{5}}, \\
a d_{f} g_{2} & =-\frac{\partial}{\partial x_{3}},
\end{aligned}
$$


with $\operatorname{dim} \Delta=5$ on an open set of 0 such that $x_{5} \neq 1$ and $v_{1}=3$ and $v_{2}=2$.

Then it is easy to check that the distribution

$$
\bar{\Delta}=\operatorname{span}\left\{g_{1}, a d_{f} g_{1}, g_{2}\right\}
$$

is involutive. Thus, the first condition of Theorem 4.1 is fulfilled. The second condition of Theorem 4.1 is obviously satisfied, because $g_{2}$ commutes with $g_{1}$ and $a d_{f} g_{1}$, i.e. $\left[g_{2}, g_{1}\right]=\left[g_{2}, a d_{f} g_{1}\right]=0$. Hence Theorem 4.1 is satisfied and the studied system can be locally transformed into the proposed 0 -flat canonical form by a local diffeomorphism.

In order to deduce the local diffeomorphism, let us calculate the codistribution of $\bar{\Delta}$. It is easy to see that

$$
\bar{\Delta}^{\perp}=\operatorname{span}\left\{d h_{1}, d h_{2}\right\},
$$

where $h_{1}=x_{3} x_{2}-x_{1}$ and $h_{2}=x_{3}$, which gives the diffeomorphism

$$
\begin{aligned}
& z_{1,1}=h_{1}, \\
& z_{2,1}=L_{f} h_{1}=\left(x_{5}-1\right) x_{2}, \\
& z_{3,1}=L_{f}^{2} h_{1}=\left(x_{5}-1\right) x_{4}, \\
& z_{1,2}=h_{2}, \\
& z_{2,2}=L_{f} h_{2}=x_{5}
\end{aligned}
$$

by which the studied system is locally transformed into the following 0 -flat form:

$$
\left\{\begin{array}{l}
\dot{z}_{1,1}=z_{2,1}, \\
\dot{z}_{2,1}=z_{3,1}+\frac{z_{2,1}}{z_{2,2}-1} u_{2}, \\
\dot{z}_{3,1}=\left(z_{2,2}-1\right) u_{1}+\frac{z_{3,1}}{z_{2,2}-1} u_{2}, \\
\dot{z}_{1,2}=z_{2,2}, \\
\dot{z}_{2,2}=u_{2} .
\end{array}\right.
$$

\section{Remark 8:}

- The specialists of linearisation by dynamic states feedback will argue that the above discussed system is linearisable, but we should remark that here we do not need the derivatives of $u$.

- If, instead of the first dynamic $\dot{x}_{1}=x_{2}+x_{4} x_{3}$ we take the same dynamic $\dot{x}_{1}=x_{4} x_{3}$ as in da Silva (2000a), then, $\Delta$ is of rank 5 on an open dense of 0 . In this case, the same flat outputs work well except that $d y_{1}\left(a d_{f}^{3} g_{1}\right) \neq 0$ and $d y_{2}\left(a d_{f}^{2} g_{2}\right) \neq 0$ on an open dense subset.

- We think that we can generalise Theorem 4.1 by assuming that distribution $\Delta$ is of dimension $n$ in a dense subset of $\mathcal{X}$ and $\bar{\Delta}$ is regular on $\mathcal{X}$.

Let us give another example to highlight the second condition of Theorem 4.1.
Example 4.4: Consider the following dynamical system:

$$
\left\{\begin{array}{l}
\dot{x}_{1}=x_{2}+\beta u_{2}+\left(\left(1+x_{3}\right) \beta+x_{5}\right) u_{3}, \\
\dot{x}_{2}=x_{3}+x_{4} u_{2}+x_{3} u_{3}, \\
\dot{x}_{3}=u_{1} \\
\dot{x}_{4}=x_{5} \\
\dot{x}_{5}=u_{2}+x_{3} u_{3} \\
\dot{x}_{6}=x_{3} x_{5} e^{x_{4}}+e^{x_{4}} u_{1}+u_{3},
\end{array}\right.
$$

where $\beta=x_{6}-x_{3} e^{x_{4}}$.

The straightforward calculation shows that the distribution $\Delta$ is generated by the following vector fields:

$$
\begin{aligned}
g_{1} & =\frac{\partial}{\partial x_{3}}+e^{x_{4}} \frac{\partial}{\partial x_{6}}, \\
a d_{f} g_{1} & =-\frac{\partial}{\partial x_{2}}, \\
a d_{f}^{2} g_{1} & =\frac{\partial}{\partial x_{1}}, \\
g_{2} & =\frac{\partial}{\partial x_{5}}+\beta \frac{\partial}{\partial x_{1}}+x_{4} \frac{\partial}{\partial x_{2}}, \\
a d_{f} g_{2} & =-\frac{\partial}{\partial x_{4}}-x_{4} \frac{\partial}{\partial x_{1}}-x_{3} e^{x_{4}} \frac{\partial}{\partial x_{6}}+x_{5} \frac{\partial}{\partial x_{2}}, \\
g_{3} & =\frac{\partial}{\partial x_{6}}+x_{3} \frac{\partial}{\partial x_{5}}+x_{3} \frac{\partial}{\partial x_{2}}+\left(x_{5}+\left(1+x_{3}\right) \beta\right) \frac{\partial}{\partial x_{1}} .
\end{aligned}
$$

Thus $v_{1}=3, v_{2}=2$ and $v_{3}=1$.

It is easy to see that

$$
\begin{gathered}
{\left[g_{2}, g_{1}\right]=0 \in \operatorname{span}\left\{g_{1}, a d_{f} g_{1}\right\}} \\
{\left[g_{3}, g_{1}\right]=-\frac{\partial}{\partial x_{2}}-\frac{\partial}{\partial x_{5}}-\beta \frac{\partial}{\partial x_{1}} \in \operatorname{span}\left\{g_{1}, a d_{f} g_{1}, g_{2}\right\} .}
\end{gathered}
$$

Thus, the second condition of Theorem 4.1 is fulfilled.

In fact, the first condition of Theorem 4.1 is also fulfilled, since the distribution

$$
\bar{\Delta}=\operatorname{span}\left\{g_{1}, a d_{f} g_{1}, g_{2}\right\}
$$

is involutive, which gives the following annihilator:

$$
\bar{\Delta}^{\perp}=\operatorname{span}\left\{d h_{1}, d h_{2}, d h_{3}\right\},
$$

where $h_{1}=x_{1}-x_{5}\left(x_{6}-x_{3} e^{x_{4}}\right), \quad h_{2}=x_{4}$ and $h_{3}=$ $x_{6}-x_{3} e^{x_{4}}$.

By setting $z_{1,1}=h_{1}, z_{1,2}=h_{2}$ and $z_{1,3}=h_{3}$, we obtain the following diffeomorphism:

$$
\begin{aligned}
& z_{1,1}=x_{1}-x_{5}\left(x_{6}-x_{3} e^{x_{4}}\right), \\
& z_{2,1}=L_{f} h_{1}=x_{2} \text { and } z_{3,1}=L_{f}^{2} h_{1}=x_{3}, \\
& z_{1,2}=h_{2}=x_{4} \text { and } z_{2,2}=L_{f} h_{2}=x_{5}, \\
& z_{1,3}=h_{3}=x_{6}-x_{3} e^{x_{4}},
\end{aligned}
$$


which locally transforms the dynamic into the following 0-flat form (4), (5):

$$
\left\{\begin{array}{l}
\dot{z}_{1,1}=z_{2,1}+z_{1,3} u_{3}, \\
\dot{z}_{2,1}=z_{3,1}+z_{1,2} u_{2}+z_{3,1} u_{3}, \\
\dot{z}_{3,1}=u_{1} \\
\dot{z}_{1,2}=z_{2.2} \\
\dot{z}_{2,2}=u_{2}+z_{3.1} u_{3}, \\
\dot{z}_{1,3}=u_{3} .
\end{array}\right.
$$

\section{Conclusion}

A triangular 0-flat canonical form was proposed in this article, which characterises a class of nonlinear dynamical systems. Sufficient and necessary geometrical conditions were given in order to deduce a local diffeomorphism with which the studied systems can be locally transformed into the proposed triangular 0-flat canonical form. Those conditions appear to be a natural generalisation of that of codimension 1 dynamical systems.

\section{Acknowledgements}

We thank Michel Fliess for all this articles and references that he gave us in the field of flatness and also for his encouragement to work in this field. We also thank all the reviewers for their useful and valuable comments in improving this article.

\section{Note}

1. Other definitions of observability indices can be found in Charlet, Lévine, and Marino (1989).

\section{References}

Anderson, R., and Ibragimov, N (1979), Lie-Bäcklund Transformations in Applications, Philadelphia: SIAM.

Bououden, S., Boutat, D., Barbot, J.P., and Kratz, F. (2009), 'A Geometrical Characterisation of a Class of 0-flat Affine Dynamical Systems', in Proceedings of IEEE ACC, St. Louis, MO, pp. 796-801.

Cartan, E. (1914), 'Sur l'Équivalence Absolue De Certains Systèmess d'Équations Différentielles et sur Certaines Familles de Courbes', Bulletin de Société Mathématique de France, 42, 12-48.

Charlet, B., Lévine, J., and Marino, R. (1989), 'On Dynamic Feedback Linearisation', Systems \& Control Letters, 13, 143-151.

da Silva, P.S.P. (2000a), 'Flatness of Nonlinear Control Systems: A Cartan-Kähler Approach', in Proceedings of Mathematical Theory of Networks and Systems, pp. 1-11. da Silva, P.S.P. (2000b), 'Flatness of Nonlinear Control Systems and Exterior Differential Systems', in Nonlinear Control in the Year 2000, eds. A. Isidori, F. LamnabhiLagarrigue and W. Respondek, Berlin: Springer-Verlag, pp. 205-227.

Delaleau, E., and da Silva, P.S.P. (1998), 'Filtrations in Feedback Synthesis: Part I - Systems and Feedbacks', Forum Mathematicum, 10, 147-174.

Descusse, J., and Moog, C. (1985), 'Decoupling with Dynamic Compensation for Strong Invertible Affine Nonlinear Systems', International Journal of Control, 42, 1387-1398.

Fliess, M., Lévine, J., Martin, P., and Rouchon, P. (1992), 'Sur les Systèmes Non-linénaires Différentiellement Plats', Comptes Rendus Mathématique, Académie des Sciences, Paris, Séries I, Mathématiques, 315, 619-624.

Fliess, M., Lévine, J., Martin, P., and Rouchon, P. (1993), 'Linéarisation par Bouclage Dynamique et Transformations de Lie-Bäcklund', Comptes Rendus Mathématique, Académie des Sciences, Paris, Series I, Mathématiques, 317, 981-986.

Fliess, M., Lévine, J., Martin, P., and Rouchon, P. (1995), 'Flatness and Defect of Non-linear Systems: Introductory Theory and Examples', International Journal of Control, 61, 1327-1361.

Fliess, M., Lévine, J., Martin, P., and Rouchon, P. (1997), 'Deux Applications de la géométrie Locale des Diffiétés', Annales de l'Institut Henri Poincare, Physics Théorique, 66, 275-292.

Fliess, M., Lévine, J., Martin, P., and Rouchon, P. (1998), 'Nonlinear Control and Diffieties, with an Application to Physics', in Secondary Calculus and Cohomological Physics (Vol. 219), eds. J. Krasilśhchik, M. Henneaux and A. Vinogradov, Providence, RI: American Mathematical Society, pp. 81-92.

Fliess, M., Lévine, J., Martin, P., and Rouchon, P. (1999a), 'A Lie-Bäcklund Approach to Equivalence and Flatness of Nonlinear Systems', IEEE Transactions on Automatic Control, 44, 922-937.

Fliess, M., Lévine, J., Martin, P., and Rouchon, P. (1999b), 'Some Open Question Related to Flat Nonlinear Systems', in Open Problems in Mathematical Systems and Control Theory, eds. V.D. Blondel, E. Sontag, M. Vidyasagar and J.C. Willems, London: Springer Verlag, pp. 99-103.

Hagenmeyer, V., and Delaleau, E. (2003), 'Robustness Analysis of Exact Feedforward Linearisation Based on Differential Fatness', Automatica, 39, 1941-1946.

Hunt, L.R., Su, R., and Meyer, G. (1983), 'Design for Multiinput Nonlinear Systems', in Differential Geometric Methods in Nonlinear Control Theory, eds. R. Brocket, R. Millmann and H.J. Sussmann, Boston, Ma: Birkhäuser, pp. 268-298.

Jakubczyk, B., and Respondek, W. (1980), 'On Linearisation of Control Systems', Bulletin de l'Academie Polonaise des Sciences, Series des Sciences Mathematiques, 28, 517-522.

Lévine, J. (2004), 'On Flatness Necessary and Sufficient Conditions', in Proceedings of NOLCOS, pp. 123-128.

Lévine, J. (2009), Analysis and Control of Nonlinear Systems: A Flatness-based Approach, Heidelberg: Springer-Verlag. 
Martin, P., Murray, R., and Rouchon, P. (1997), 'Flat Systems', in European Control Conference, pp. 211264.

Martin, P., and Rouchon, P. (1995a), 'Any (Controllable) Driftless System with 3 Inputs and 5 States is Flat', Systems \& Control Letters, 25, 167-173.

Martin, P., and Rouchon, P. (1995b), 'Any (controllable) Driftless System with $m$ Inputs and $m+2$ States is Flat', in Proceedings of the IEEE Conference on Decision and Control, pp. 2886-2891.

Pomet, J.B. (1995a), 'A Differential Geometric Setting for Dynamic Equivalence and Dynamic Linearization', in Geometry in Nonlinear Control and Differential inclusions, eds. B. Jackubczyk, W. Respondek and T. Rzezuchowski, Warsaw: Banach Center Publications, pp. 319-339.

Pomet, J.B. (1995b), 'On Dynamic Feedback Linearization of Four-dimensional Affine Control Systems with Two Inputs', Technical, Memorandum RR-2751, I.N.R.I.A., Books and Monographs, http://www.emath.fr/

Rotella, F. and Zambettakis, I. (2007), 'Commande des Systèmes par Platitude', Techniques de l'Ingènieurs, http:// www.techniques-ingenieur.fr, 57450.

Rouchon, P. (1995), 'Necessary Condition and Genericity of Dynamic Feedback Linearisation', Journal of Mathematical Systems, Estimation, and Control, 5, 345-358.

Rudolph, J. (1995), 'Well-formed Dynamics Under Quasistatic State Feedback', in Geometry in Nonlinear Control and Differential Inclusions, eds. B. Jackubczyk, W. Respondek and T. Rzezuchowski, Warsaw: Banach Center Publications, pp. 349-360.

Schlacher, K., and Schöberl, M. (2007), 'Construction of Flat Outputs by Reduction and Elimination', in Proceedings of NOLCOS, pp. 666-671.
Shadwick, W. (1990), 'Absolute Equivalence and Dynamic Feedback Linearisation', Systems and Control Letters, 15, $35-39$.

Shadwick, W., and Sluis, W. (1994), 'Dynamic Feedback for Classical Geometries', in Differential Geometry and Mathematical Physics, eds. J.K. Beem and K.L. Duggal, Providence, RI: American Mathematical Society, pp. 207-213.

Silveira, H. (2009), 'Formas Triangulares para Sistemas não Lineares com duas Entradas e Controle de Sistemas sem Arrasto em SU(n) com Aplicações em Mecânica Quântica', Ph.D. Thesis, Universidade de São Paulo, Brazil.

Silveira, H., and da Silva, P.S.P. (2007), 'A Triangular Form for Flat Nonlinear Systems with Two Controls and Five or Less States', in Proceedings of the 3rd IFAC Symposium on System, Structure and Control, pp. 127-132.

Singh, S. (1981), 'A Modified Algorithm for Invertibility in Nonlinear Systems', IEEE Transactions on Automatic Control, 26, 595-598.

Sira-Ramirez, H., and Agrawal, S. (2004), Differentially Flat Systems, New York: Marcel Dekker.

Sluis, W. (1993), 'A Necessary Condition for Dynamic Feedback Linearisation', Systems and Control Letters, 21, 277-283.

Sluis, W., and Tilbury, D. (1996), 'A Bound on the Number of Integrators Needed to Linearise a Control System', Systems and Control Letters, 29, 43-50.

Tilbury, D., Murray, R., and Sastry, S. (1995), 'Trajectory Generation for the n-trailer Problem using Goursat Normal Form', IEEE Transactions on Automatic Control, 40, 802-819.

van Nieuwstadt, M., Rathinam, M., and Murray, R. (1998), 'Differential Flatness and Absolute Equivalence of Nonlinear Control Systems', SIAM Journal of Control Optimization, 36, 1225-1239. 